\title{
Cost-effectiveness analyses for mirtazapine and sertraline in dementia: randomised controlled trial
}

Renee Romeo, Martin Knapp, Jennifer Hellier, Michael Dewey, Clive Ballard, Robert Baldwin, Peter Bentham, Alistair Burns, Chris Fox, Clive Holmes, Cornelius Katona, Claire Lawton, James Lindesay, Gill Livingston, Niall McCrae, Esme Moniz-Cook, Joanna Murray, Shirley Nurock, John O'Brien, Michaela Poppe, Alan Thomas, Rebecca Walwyn, Kenneth Wilson and Sube Banerjee

\section{Background}

Depression is a common and costly comorbidity in dementia. There are very few data on the cost-effectiveness of antidepressants for depression in dementia and their effects on carer outcomes.

\section{Aims}

To evaluate the cost-effectiveness of sertraline and mirtazapine compared with placebo for depression in dementia.

\section{Method}

A pragmatic, multicentre, randomised placebo-controlled tria with a parallel cost-effectiveness analysis (trial registration: ISRCTN88882979 and EudraCT 2006-000105-38). The primary cost-effectiveness analysis compared differences in treatment costs for patients receiving sertraline, mirtazapine or placebo with differences in effectiveness measured by the primary outcome, total Cornell Scale for Depression in Dementia (CSDD) score, over two time periods: 0-13 weeks and 0-39 weeks. The secondary evaluation was a cost-utility analysis using quality-adjusted life years (QALYS) computed from the Euro-Qual (EQ-5D) and societal weights over those same periods.

\section{Results}

There were 339 participants randomised and 326 with costs data (111 placebo, 107 sertraline, 108 mirtazapine). For the primary outcome, decrease in depression, mirtazapine and sertraline were not cost-effective compared with placebo. However, examining secondary outcomes, the time spent by unpaid carers caring for participants in the mirtazapine group was almost half that for patients receiving placebo $(6.74 \mathrm{~V}$. 12.27 hours per week) or sertraline (6.74 v. 12.32 hours per week). Informal care costs over 39 weeks were $\mathrm{f} 1510$ and f1522 less for the mirtazapine group compared with placebo and sertraline respectively.

\section{Conclusions}

In terms of reducing depression, mirtazapine and sertraline were not cost-effective for treating depression in dementia. However, mirtazapine does appear likely to have been costeffective if costing includes the impact on unpaid carers and with quality of life included in the outcome. Unpaid (family) carer costs were lower with mirtazapine than sertraline or placebo. This may have been mediated via the putative ability of mirtazapine to ameliorate sleep disturbances and anxiety. Given the priority and the potential value of supporting family carers of people with dementia, further research is warranted to investigate the potential of mirtazapine to help with behavioural and psychological symptoms in dementia and in supporting carers.

\section{Declaration of interest}

S.B., M.D., C.B., R.B., P.B., C.F., C.H., C.K., M.K., C.L., J.L., G.L., E.M.-C., J.M., J.O'B., A.T., K.W. and A.B. have received consultancy fees, speakers' fees, research funding or educational support to attend conferences from pharmaceutical companies involved in the manufacture of antidepressants and antidementia drugs.
Dementia is one of the greatest health and social care challenges we face, with costs set to increase substantially as the numbers of people with dementia rise. The estimated global costs of dementia, US $\$ 604$ billion ( $£ 386$ billion) in 2010 , are projected to increase by $85 \%$ by $2030 .{ }^{1}$ In England alone, the cost of longterm care of older people with dementia is set to increase from

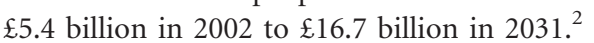

Depression is a common and important comorbidity in dementia. Prevalence estimates vary from 5 to $40 \%$ depending on sampling and definition. ${ }^{3-5}$ Depression in dementia is a risk factor for increased carer distress, disability, suicide and mortality, and is also associated with a high level of use of medical in-patient beds. ${ }^{6}$ Treating depression in dementia is therefore a clinical priority with the potential to improve the well-being, quality of life and level of function of people with dementia; it might also have an effect on costs.

However, there is only weak evidence on the effectiveness of antidepressants for depression in dementia, ${ }^{7}$ and no cost-effectiveness evidence on the treatment of depression in dementia. ${ }^{8}$ Despite this, antidepressants are frequently used for treating depression in dementia. The Health Technology Assessment Study of the Use of Antidepressants for Depression in Dementia (HTA-SADD) trial was designed to investigate the clinical effectiveness and cost-effectiveness of the two most commonly used classes of antidepressants compared with placebo. We have published our analyses of clinical effectiveness ${ }^{9}$ and reported that those randomised to sertraline or mirtazapine did no better than those receiving placebo. Here we report on the co-primary aim: to examine the cost-effectiveness of sertraline and mirtazapine compared with placebo over 13 weeks and 39 weeks in people with depression and dementia.

\section{Method}

\section{Research setting}

We have published the details of the HTA-SADD trial method and results elsewhere. ${ }^{9}$ To summarise, the trial involved people with probable or possible dementia of the Alzheimer's type according to NINCDS-ADRDA (National Institute of Neurological and 
Communicative Disorders and Stroke and the Alzheimer's Disease and Related Disorders Association) criteria ${ }^{10}$ and depression (defined as a Cornell Scale for Depression in Dementia (CSDD) $)^{11}$ score of over 7) referred to old age psychiatric services in nine English sites: Birmingham, Cambridge, Leicester, Liverpool, Manchester, Newcastle, North London, Southampton and South London. Ethical approval was obtained from the North West 7 (Greater Manchester) Ethics Committee.

\section{Sample size}

A sample size of 507 was calculated to provide $90 \%$ power to detect a two-point CSDD difference (s.d. $=5$; standardised effect-size $\quad(\mathrm{SES})=0.4)$ for 13 -week sertraline/placebo and mirtazapine/placebo comparisons, and $86 \%$ power at 39 weeks. This was revised during the trial because of slower than forecasted recruitment to $339 .^{9}$

\section{Randomisation}

After baseline assessment and consent, participants were randomised to three groups: sertraline, mirtazapine and placebo (trial registration: ISRCTN88882979 and EudraCT 2006-000105-38). All participants also received normal clinical care. The target doses were $150 \mathrm{mg}$ sertraline or $45 \mathrm{mg}$ mirtazapine daily with titration over 8 weeks. Thereafter it was open to clinicians to adjust the dose. The Clinical Trials Unit at the Institute of Psychiatry independently undertook treatment allocation.

\section{Economic evaluation}

The primary economic evaluation was a cost-effectiveness analysis comparing differences in treatment costs for patients receiving sertraline, mirtazapine or placebo with differences in effectiveness as measured by the primary outcome, total CSDD score ${ }^{11}$ over two time periods: $0-13$ weeks and $0-39$ weeks. The secondary analysis was a cost-utility analysis using quality-adjusted life years (QALYs) computed from the Euro-Qual (EQ-5D) ${ }^{12}$ and societal weights (obtained from data from a sample of the general public to aggregate items into an overall EQ-5D score ${ }^{13}$ over those same periods. Both the primary and secondary economic evaluations were undertaken from two perspectives: (a) health and social care agencies; and (b) health and social care agencies and unpaid carers.

\section{Resource use}

Resource-use data for each person were collected over a retrospective period of 6 months before randomisation. At 13 weeks, follow-up data were collected retrospectively for a 3-month period and at 39 weeks for a retrospective period of 6 months. Services and support received by the study participants were recorded on a resource-use questionnaire adapted from the Client Service Receipt Inventory (CSRI), ${ }^{14}$ including in-patient stays, out-patient attendances, day hospital treatment, visits to social clubs, meals at lunch clubs, day care visits and hours spent in contact with community-based professionals. The study also collected data on volunteer support, befriending and telephone care-line support, and also on unpaid support provided by friends and relatives. Contacts made with voluntary workers and support provided by friends and relatives were also measured in hours of care support time. The prescribed daily doses for the medications were calculated from the trial medication log, and prescribing periods were weighted to the changing dose regime.

\section{Unit costs}

All unit costs were estimated at 2009/2010 prices and were collected from sources in the public domain (Table 1). Costs per unit of measurement for each type of service (such as per in-patient day, per appointment, per attendance, per visit or per contact with health and community-based professionals including voluntary services) were taken from a widely used compendium. ${ }^{15}$ The National Health Service Schedule of Reference Costs was used to estimate the cost of out-patient attendances. ${ }^{16}$ The unit cost of medication was obtained from the British National Formulary. ${ }^{17}$

We collected information from each study participant's main carer on time they spent providing unpaid care and support, and time spent by friends or relatives regularly providing help for the trial participant. We asked respondents to estimate the hours of unpaid care and support from all such sources in an average or typical week. Opportunity costs were attached to these hours using, first, an estimate of replacement cost (the unit cost of a paid local authority home care worker ${ }^{15}$ ) and, second, the cost of lost employment (gross hourly wage of a carer in paid employment; zero for a carer not in paid employment).

\section{Cost estimation}

Data on resource use from the CSRI and medication prescribed from the medication logs were combined with unit cost data to

\begin{tabular}{|c|c|c|}
\hline Service & Unit cost $(f)$ & Source \\
\hline In-patient (bed days) & 299 & 15,26 \\
\hline Day hospital (attendance) & 50-205 & $15,16,26$ \\
\hline Out-patient (appointment) & $21-165$ & 16 \\
\hline Accident and emergency (attendance) & $37-97$ & 15 \\
\hline General practitioner (per surgery consultation) & 28 & 15 \\
\hline Geriatrician (min) & 1.83 & 15 \\
\hline Nurse $(\min )^{a}$ & $0.43-0.52$ & 15 \\
\hline Occupational therapist (min) & 0.65 & 15 \\
\hline Community psychiatrist (min) & 1.83 & 15 \\
\hline Counsellor (min) & 0.57 & 15 \\
\hline Psychologist (min) & 1.20 & 15 \\
\hline Chiropodist (contact) & 0.37 & 15 \\
\hline Social worker (min) & 0.67 & 15 \\
\hline Care manager (min) & 0.82 & 15 \\
\hline Home care worker/care attendant (min) & 0.35 & 15 \\
\hline Sitting scheme (min) & 0.45 & 15 \\
\hline Self-help group (min) & 0.57 & 15 \\
\hline Meals on wheels (meal) & 4.8 & $\mathrm{~b}$ \\
\hline Dentist (min) & 2.90 & 16 \\
\hline Optician (min) & 0.48 & c \\
\hline Day care (day) & $42-66$ & 15 \\
\hline Lunch club (meal) & 7 & d \\
\hline Social club (session) & 5 & e \\
\hline \multicolumn{3}{|c|}{$\begin{array}{l}\text { a. Practice nurse, district nurse health visitor, community psychiatric nurse, cardiac } \\
\text { nurse, incontinence nurse. } \\
\text { b. http://www.ic.nhs.uk/webfiles/publications/009_Social_Care/pss0910expfinal/ } \\
\text { pss0910updateOct2011/Personal_Social_Services_Expenditure_Report_2009_10.pdf } \\
\text { c. New calculation: there is a recommended fee payable to ophthalmic medical } \\
\text { practitioners who administer sight tests, although optometrists undertake most tests. } \\
\text { Optometrist salaries vary depending on practice setting (private or hospital or } \\
\text { combination of the two). Typical salaries in private practice based on salary data } \\
\text { collected June } 2009 \text { (http://Www.prospects.ac.uk/optometrist_salary.htm) ranged from } \\
\text { f19500 to f28 } 000 \text {. In hospital settings, optometrist salaries are usually covered by } \\
\text { the Agenda for Change pay scale. Average salary for private practice was used. } \\
\text { Cost per hour was estimated based on } 41 \text { weeks per annum, } 38 \text { hours per week. } \\
\text { d. http://cash-online.org.uk/content/1/6/3/; uprated using the Consumer Price Index } \\
\text { (CPI). } \\
\text { e. Cost of adult social club at } 2004 / 05 \text { uprated using the pay and prices inflator. }{ }^{15}\end{array}$} \\
\hline
\end{tabular}


estimate total costs for each participant in the trial. Three main categories of costs were analysed: medication costs, aggregated health and social care costs (primary care and hospital-based visits and community-based contacts) and (opportunity) cost of time spent caregiving by relatives and friends. Costs were categorised in this way to facilitate comparison of costs alongside measures of effectiveness from the various study perspectives. The costs of services and support used by patients were derived by combining medication, health and social care resource utilisation data with unit costs. Costs were calculated for the periods $0-13$ weeks and 0-39 weeks.

\section{Statistical analysis}

An 'intention-to-treat' analysis was carried out to preserve the unbiased distribution of factors in the groups produced by randomisation. Missing resource-use data were singly imputed. If there was no report on the use of a particular resource, we assumed that it was not used. If participants reported on a resource but not the quantity used, we imputed this amount from within treatment-group means for participants with data for that item at the same assessment point.

Health and social care costs for 0-13 weeks and 0-39 weeks (and health/social care and costs of unpaid carer costs for the parallel analysis from the broader perspective for the same time periods) were regressed in turn on treatment allocation, baseline cost, baseline CSDD and centre. To mitigate the effects of skewness, non-parametric bootstrapping methods were used to estimate $95 \%$ confidence intervals (CIs) for mean costs. Where the bias-corrected 95\% CIs of between-group change scores excluded zero, they could be judged to be significant at $P=0.05$ or lower.

Estimates of bootstrapped mean cost and effectiveness were used to estimate an incremental cost-effectiveness ratio (ICER) for each analysis. The ICER for each replication was calculated as:

$$
\left(\operatorname{cost}_{\mathrm{b}}-\operatorname{cost}_{\mathrm{a}}\right) /\left(\text { effect }_{\mathrm{b}}-\text { effect }_{\mathrm{a}}\right),
$$

which summarises the cost difference between two treatments per incremental difference in the outcome (CSDD and EQ-5D in turn). The EQ-5D was measured directly from patients - as recommended by National Institute for Health and Clinical Excellence (NICE) guidelines ${ }^{18}$ - and weighted by a valuation of changes in quality of life reported from UK population data. ${ }^{13}$ Health effects were then expressed in terms of QALYs. The ratio statistic compared the treatments in terms of observed differences in costs and effects, regardless of whether those differences were statistically significant.

Uncertainty about the cost and effectiveness estimates was addressed by plotting cost-effectiveness acceptability curves (CEAC). A CEAC was used to assess trade-offs between costs and outcomes, showing the likelihood of each of the two medications in turn being seen as cost-effective relative to the other or relative to placebo, given different (implicit monetary) values placed on incremental outcome improvements. In this net-benefit approach, monetary values of incremental effects and incremental costs for each case are combined, and the net benefit derived as:

$$
\mathrm{NB}=\lambda \times\left(\text { effect }_{\mathrm{b}}-\mathrm{effect}_{\mathrm{a}}\right)-\left(\operatorname{cost}_{\mathrm{b}}-\operatorname{cost}_{\mathrm{a}}\right),
$$

where: $a$ is the comparator, $b$ is drug treatment, NB is net benefit, and $\lambda$ is willingness to pay for a unit improvement in CSDDdepression severity score (primary evaluation) or an additional QALY (secondary evaluation). The impact on costs given uncertainty around the value attached to informal care inputs was assessed in one-dimensional sensitivity analysis. All analyses were completed in Stata (version 11) and SPSS 17 on Windows.

\section{Results}

\section{Baseline comparisons}

Overall, 339 participants were recruited to the trial. At baseline, full service-use data were available for 326 participants (111 placebo, 107 sertraline, 108 mirtazapine). At 13 weeks, economic data were available for 97 (87\%) participants in the placebo group, $78(73 \%)$ in the sertraline group and $88(81 \%)$ in the mirtazapine group. By 39 weeks there were economic data on $84(76 \%)$ participants in the placebo group, 69 (64\%) in the sertraline group and $78(72 \%)$ in the mirtazapine group. This drop-out level is comparable with other trials in the area.

\section{Service use and support}

Contacts made by study participants with services and support over weeks $0-13$ and $0-39$ are shown in Tables 2 and 3 respectively. In the $0-13$-week period (Table 2), there were no differences in service use between the treatment groups reaching statistical significance at the $5 \%$ level. However, taking the whole 0-39 week period (Table 3), it was striking that the mean number of hours per week spent by unpaid carers caring for patients in the placebo-treated group and the sertraline group were almost twice that for patients in the mirtazapine-treated group. This difference in unpaid carer time between the placebo and mirtazapine-treated group was statistically significant at the $5 \%$ level.

\section{Outcomes}

At 39 weeks, the difference in mean CSDD score between placebo and sertraline was 0.05 ( $95 \% \mathrm{CI}-1.83$ to 1.67$)$; between placebo and mirtazapine was -0.80 (95\% CI -2.55 to 1.21$)$; and between mirtazapine and sertraline was -0.9 ( $95 \% \mathrm{CI}-1.10$ to 2.73$)$. The secondary measure of outcome was QALY gain at 39 weeks; the mean difference between placebo and sertraline was 0.03 ( $95 \% \mathrm{CI}-0.09$ to $0.03)$; between placebo and mirtazapine was 0.05 (95\% CI -0.10 to $0.01)$; and between mirtazapine and sertraline was $0.02(95 \% \mathrm{CI}$ -0.03 to 0.07 ). There were no statistically significant differences in either the primary or secondary measure of outcome between groups at 39 weeks (or at 13 weeks) (Table 4).

\section{Costs}

Daily medication costs for sertraline $50 \mathrm{mg}$ of $\mathfrak{L}_{0.05}$ and mirtazapine $15 \mathrm{mg}$ of $\mathfrak{E} 0.23$ were applied (mean cost of medication per person: $\mathfrak{E 7}$ (95\% CI 6-8) and $£ 37$ (95\% CI 32-41)). Mean total costs over 0-13 weeks and 0-39 weeks are detailed in Table 4. Pair-wise comparisons were made between the two antidepressants and placebo using regression analysis and bootstrapping. There were no statistically significant differences between the groups in either of the time periods, either when health and social care service costs only were considered, or when health and social care services and unpaid carer costs were summed. After adjustment for baseline costs, CSDD score at baseline and site, there were no statistically significant differences in health and social care costs - or in health/social care and unpaid carer costs - in any pair-wise comparison in either time period.

Unpaid carer costs exceeded health and social care costs by a factor of 1.2 to 1.7. Including these unpaid carer costs results in a change in the ranking of total costs, with mirtazapine being the least expensive of all treatments in both periods.

\section{Cost-effectiveness}

As noted earlier, the primary economic evaluation focused on CSDD as the outcome over, first, the period $0-13$ weeks after randomisation, and, second, the period 0-39 weeks after 


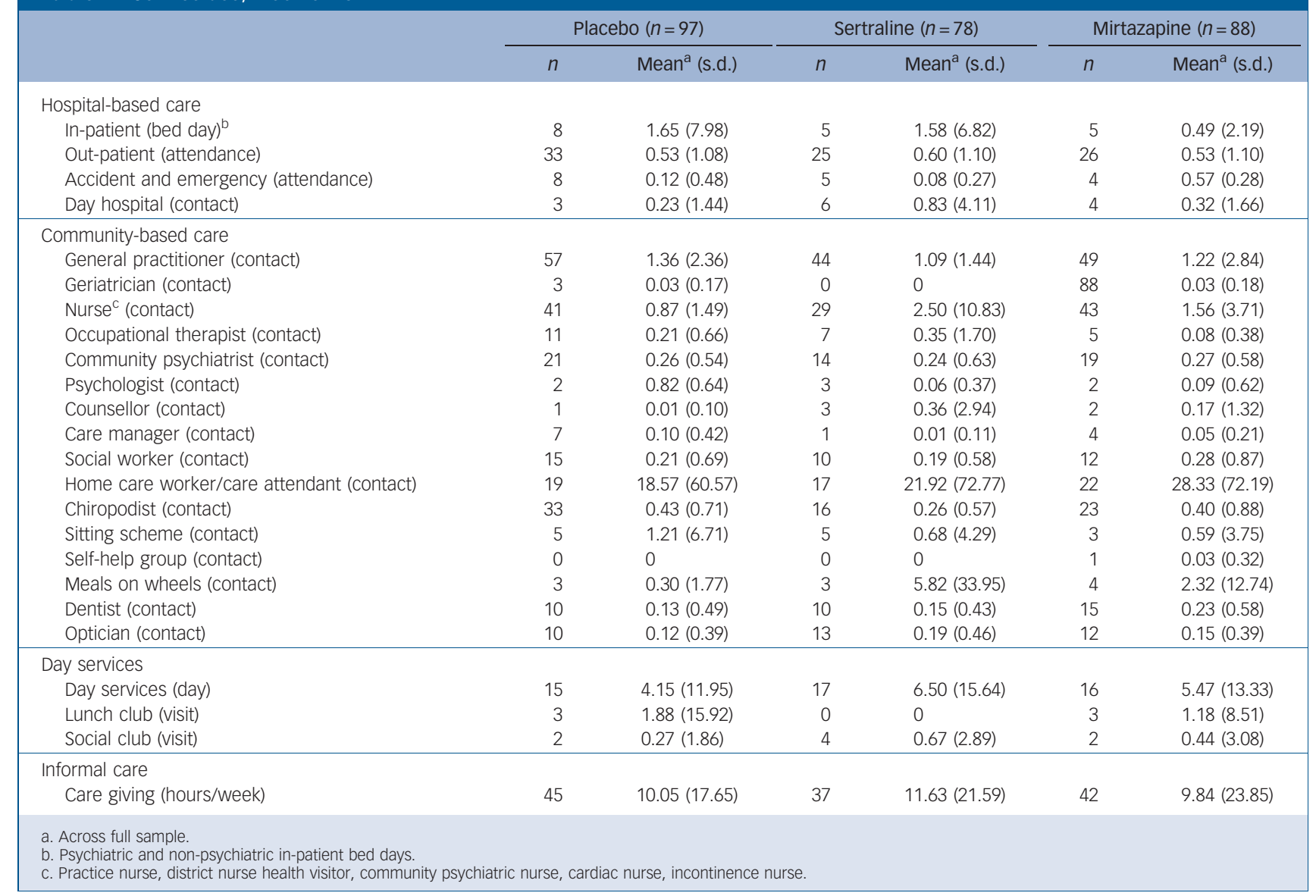

\section{Table 3 Service use, week 0-39}

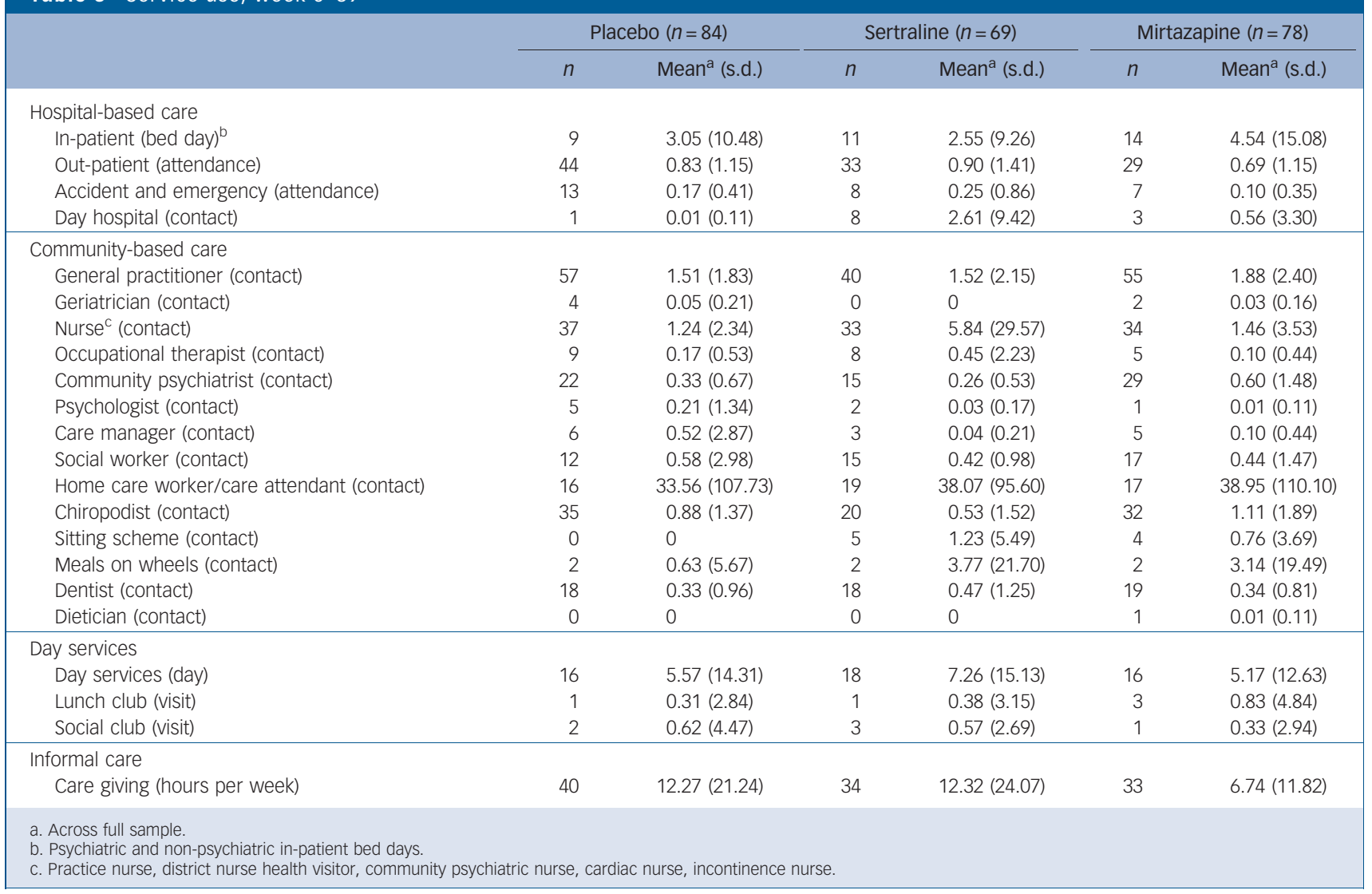




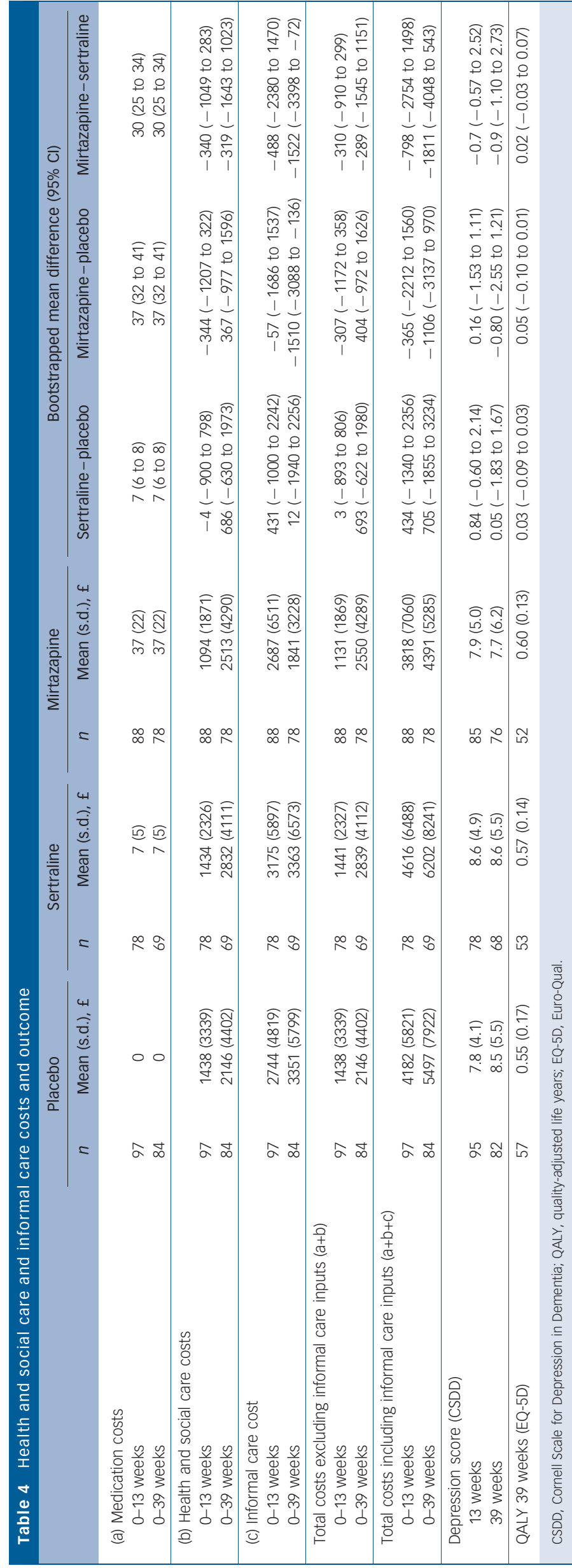

randomisation. The secondary evaluation focused on QALYs computed from the EQ-5D and societal weights over the same periods. Data used in the estimation of the ICERs are shown in Table 5. As noted earlier, there were no statistically significant differences in CSDD scores or QALYs in any of the pair-wise comparisons. There were also no significant pair-wise differences in costs from either perspective between the treatment groups.

Probability estimates were plotted for a range of implicit monetary values attached to improvements in depression score and QALY gain. We are not aware of any studies that have attached monetary values to incremental changes in CSDD to provide any guidance on the appropriate range of willingnessto-pay values.

In Fig. 1, we see that mirtazapine had a low likelihood (around $30 \%$ ) of being more cost-effective than placebo if society were not willing to pay anything for a unit improvement in the CSDD depression score. The likelihood of cost-effectiveness rose to $80 \%$ if society were willing to pay $£ 5000$ for a unit improvement in CSDD score, and stayed at $80 \%$ over values of willingness to pay for an improvement in CSDD score up to $£ 30000$.

Figure 2 shows the CEACs from the secondary economic evaluation, where health and social care costs including unpaid carer inputs and health and social care costs excluding unpaid carer inputs were considered alongside QALYs in turn. It suggests that from a health and social care perspective mirtazapine was

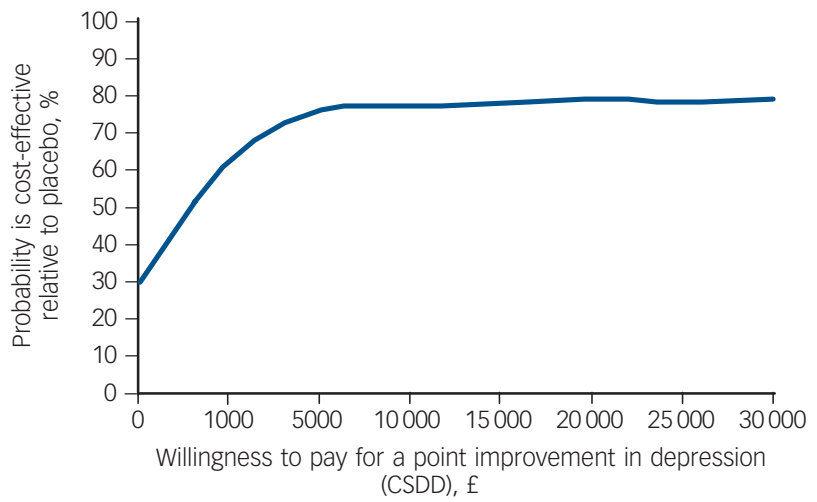

Fig. 1 Probability that mirtazapine is cost-effective compared with placebo: health and social care costs and Cornell Scale for Depression in Dementia score (CSDD) over 39 weeks.

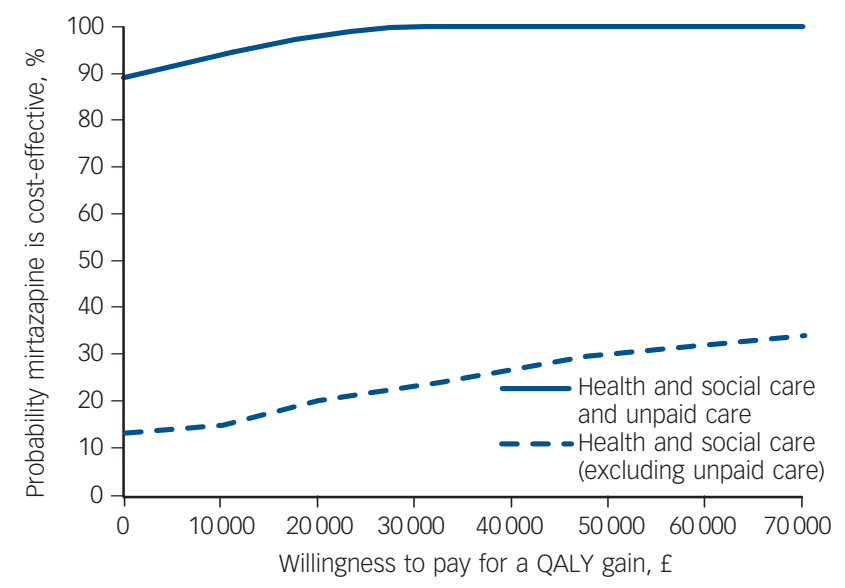

Fig. 2 Probability that mirtazapine is cost-effective relative to placebo: health and social care costs and quality-adjusted life years (QALYS) over 39 weeks. 


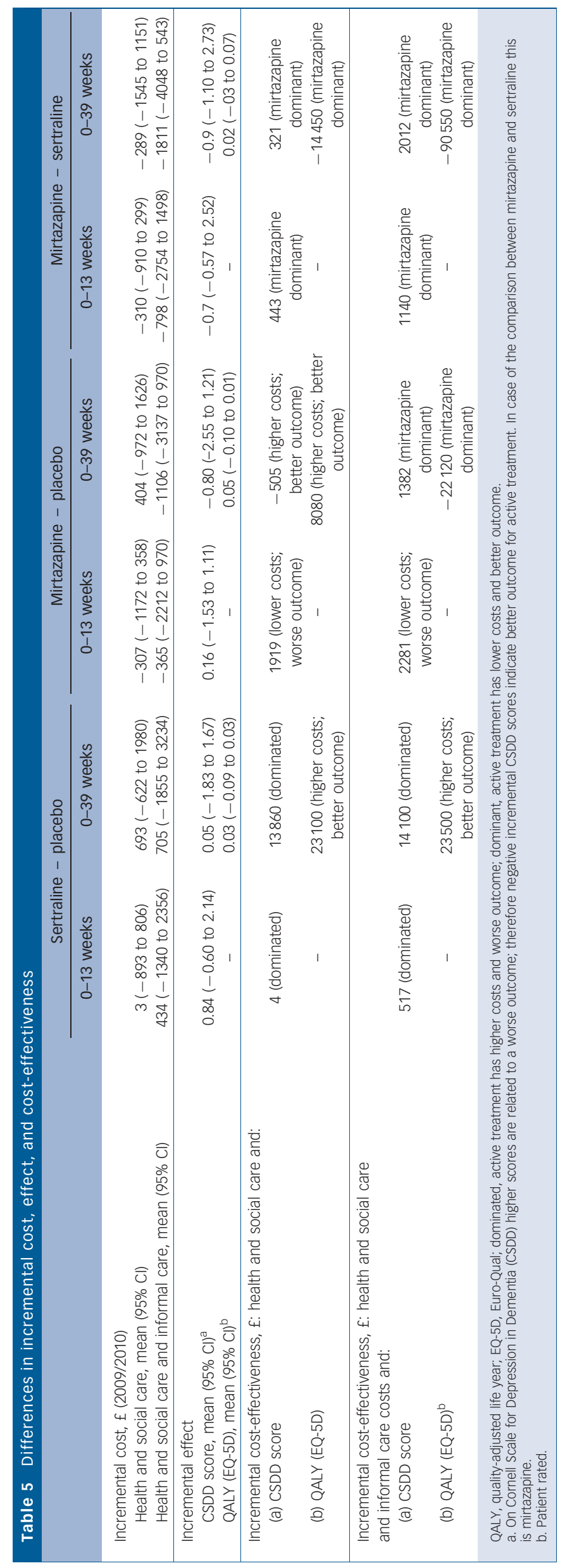

$14 \%$ likely to be more cost-effective than placebo even if society were willing to pay nothing for a QALY gain. This probability increased to over $20 \%$ for a willingness to pay $£ 30000$ for a QALY gain. However, when health and social care costs including unpaid carer inputs were considered alongside QALYs, mirtazapine was $89 \%$ likely to be more cost-effective than placebo even if society

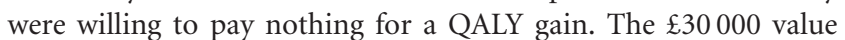
is important as it is the threshold often associated with positive decisions on health technologies taken by NICE.

We assessed uncertainty around parameter estimates included in the cost analysis. For the main analyses, unpaid carer costs were based on the hourly cost of a home care worker. This hourly value for the caregiving inputs by friends and family was replaced in sensitivity analysis by the gross hourly wage of a carer in paid employment and zero for a carer not in paid employment. Using these alternative values of caregiver time inputs did not alter the findings (Table 6).

\section{Discussion}

As far as we are aware this is the first study to explore the costeffectiveness of mirtazapine and sertraline in treating depression in dementia. Our results show that mirtazapine and sertraline are not cost-effective compared with placebo as a treatment for depression in dementia when looking at the primary outcome of change in depressive symptoms (i.e. neither of the antidepressants reduced CSDD score more than placebo).

However, mirtazapine did halve unpaid carer time and therefore carer costs. So, when costs were considered alongside QALY gains, a different picture emerged. Mirtazapine had the highest likelihood of cost-effectiveness compared with sertraline and placebo. Some previous studies have similarly reported a cost-effectiveness advantage when using the QALY as the outcome measure even though there was no discernible cost-effectiveness difference on the primary symptom measure, including studies of dementia ${ }^{19}$ and depression. ${ }^{20}$ The probabilities of cost-effectiveness at a willingness-to-pay value of $£ 30000$ per QALY and under are relevant given that NICE uses this value as a threshold to guide decisions about whether or not to recommend health technologies. ${ }^{21}$

We considered possible reasons for the finding that mirtazapine treatment had a good chance of being cost-effective compared with placebo or sertraline when the outcome under consideration is QALY. The trend towards lower incremental costs for mirtazapine was driven by the statistically significantly lower unpaid carer inputs. The small improvements in quality of life for mirtazapine relative to the other treatments also contributed to the costeffectiveness result, and can perhaps be mediated plausibly via the putative ability of mirtazapine to ameliorate sleep disturbances and anxiety. ${ }^{22,23}$ Improvements in sleep could potentially improve life quality and therefore patient-reported EQ-5D scores; they could also release carer time directly and so ameliorate an important source of carer distress. ${ }^{24}$ In this way mirtazapine might have a general effect, beneficial for both the patient and the carer, without exerting a specific antidepressant effect. The extent to which this is generalisable to other antidepressants is not clear from our study. The potential positive effects of mirtazapine act more in the realm of general behavioural and psychological symptoms in dementia than depression per se. It is possible that a positive effect on sleep, anxiety or agitation in the person with dementia might result in relief, not only for the person with dementia but also the carer, in terms of hours of care needed. However, it is also important to note that complex ethical issues are raised potentially when treatment is given to patients for the benefit of their carers. 
Table 6 Sensitivity analysis

\begin{tabular}{|c|c|c|c|c|c|}
\hline & \multicolumn{3}{|c|}{ Mean (s.d.) } & \multicolumn{2}{|c|}{ Mean difference $(95 \% \mathrm{Cl})$} \\
\hline & Placebo & Sertraline & Mirtazapine & Sertraline - placebo & Mirtazapine - placebo \\
\hline Main analysis: 0-13 weeks (total cost including informal care) & $4182(5821)$ & $4616(6488)$ & $3818(7060)$ & $434(-1340$ to 2356$)$ & $-365(-2212$ to 1560$)$ \\
\hline (a) Applying gross wage for informal care inputs & $3368(4769)$ & $3663(5008)$ & $3592(5461)$ & $322(-1081$ to 1797$)$ & $-353(-1778$ to 1087$)$ \\
\hline Main analysis: 0-39 weeks (total cost including informal care) & $5497(7922)$ & $6202(8241)$ & $4391(5285)$ & $705(-1855$ to 3234$)$ & $-1106(-3137$ to 970$)$ \\
\hline (a) Applying gross wage for informal care inputs & $4476(6512)$ & $5177(6574)$ & $3830(4777)$ & $702(-1313$ to 2751$)$ & $-645(-2415$ to 986$)$ \\
\hline
\end{tabular}

\section{Strengths and limitations of the study}

This is the first randomised controlled trial with an economic evaluation of pharmacotherapy for people with dementia and depression, based on individual patient data, and so fills an important evidence gap. Further, its findings are likely to have a wide application because of the broad nature of the study group, in terms of both the range of depressive symptoms and the severity of dementia. The study included individuals with probable and possible Alzheimer's. This group is close to the population found in clinical practice where there is often a vascular component to dementia. However, we would limit generalisability of the study's findings to those with Alzheimer's disease and mixed dementia only, and not to other subtypes such as vascular dementia, dementia with Lewy bodies or frontotemporal dementia.

There were some incomplete data from the CSRI, which was to be expected given the size and spread of the sample and the comprehensive nature of the service-use data-collection exercise. It would not have been feasible to collect these data from alternative sources. Missing responses were therefore assigned a value by imputation to make efficient use of the data provided.

The computation of unpaid carer costs is always difficult and we built estimates in this study on two different assumptions about unit cost, and did not reach different conclusions about cost-effectiveness. However, we were still reliant on carers' selfreported numbers of hours spent providing support to trial participants, and this is an aspect of evaluation that needs more attention to ensure sufficient accuracy.

\section{Implications}

Following the current policy-making stance of focusing on health and social care costs, the findings reported here suggest that using these drugs (relative to placebo) for treating depression in dementia is unlikely to be cost-effective if a narrow focus on depression score is adopted. However, treatment with mirtazapine appears likely to be cost-effective if a broad cost-perspective is used (to include the impact on health and social care services and the impact on unpaid carers) and if a broader approach is taken to outcome measurement to look at health-related quality of life. Family carers are a vital resource in dementia care and their support is an explicit policy priority in England. ${ }^{25}$ Given the priority and the potential value of supporting family carers of people with dementia, further research is warranted to investigate the potential of mirtazapine to help with behavioural and psychological symptoms in dementia and in supporting carers.

\section{Funding}

This trial was funded by the UK National Institute of Health Research Health Technology Assessment Programme.

\section{Acknowledgements}

We would like to thank all the participants and carers that gave their time to be part of this study. We thank Pfizer for their kind donation of the sertraline and sertraline placebo for this trial. Thanks are due to the members of the HTA-SADD Data Monitoring and Ethics Committee and the HTA-SADD Trial Steering Committee - Peter Connelly (chair of the Data Monitoring and Ethics Committee), Robin Jacoby (chair of the Trial Steering Committee), Rowan Harwood, Cornelius Kelly, Angela Clayton-Turner, Craig Ritchie and Ed Juszczak. We thank the Alzheimer's Society for providing patient and public involvement support into We thank the Alzheimer's Society for providing patient and public involvement support into
the study. We are grateful for the practical help provided by the NIHR Mental Health the study. We are grateful for the practical help provided by the NIHR Mental Health
Research Network (MHRN) and Dementia and Neurodegenerative Disease Research Network (DeNDRON).

Renee Romeo, PhD, Health Services and Population Research Department, Institute of Psychiatry, King's College London; Martin Knapp, PhD, Health Services and Population Research Department, Institute of Psychiatry, King's College London and Personal Social Services Research Unit, London School of Economics and Political Science; Jennifer Hellier, PhD, Mental Health and Neuroscience Clinical Trials Unit, King's College London; Michael Dewey, PhD, Health Services and Population Research Department, Institute of Psychiatry, King's College London; Clive Ballard Research Department, Institute of Psychiatry, King's College London; Clive Ba
MD, Wolfson Centre for Age-Related Disease, King's College London; Robert Baldwin, MD, Department of Community Based Medicine, University of Manchester Peter Bentham, MRCPsych, Department of Psychiatry, University of Birmingham; Alistair Burns, MD, Department of Community Based Medicine, University of Manchester; Chris Fox, MD, School of Medicine, University of East Anglia, Norwich; Clive Holmes, PhD, Clinical and Experimental Sciences Division, University of Southampton; Cornelius Katona, MD, Department of Mental Health Sciences, Southampton; Cornelius Katona, MD, Department of Mental Health Sciences,
University College London; Claire Lawton, FRCPsych, Department of Psychiatry, University of Cambridge; James Lindesay, DM, Department of Psychiatry, University of Leicester; Gill Livingston, MD, Department of Mental Health Sciences, University College London; Niall Mccrae, PhD, Health Services and Population Research Department, Institute of Psychiatry, King's College London; Esme Moniz-Cook, PhD, Institute of Rehabilitation, Hull York Medical School, Hull; Joanna Murray, MA, Health Services and Population Research Department, Institute of Psychiatry, King's College London; Shirley Nurock, MSc, Alzheimer's Society, Research Network Volunteer; John O'Brien, DM, Institute for Ageing and Health, Newcastle University; Michaela Poppe, PhD, Health Services and Population Research Department, Institute of Psychiatry, King's College London; Alan Thomas, PhD, Institute for Ageing and Health, Newcastle University; Rebecca Walwyn, PhD, Mental Health and Neuroscience Clinical Trials Unit, King's College London; Kenneth Wilson, MD, Department of Psychiatry, Liverpool University; Sube Banerjee, MD, Centre for Dementia Studies, Brighton and Sussex Medical School, Brighton, UK.

Correspondence: Sube Banerjee, Brighton \& Sussex Medical School, BSMS Teaching Building, University of Sussex, Brighton, East Sussex BN1 9PX, UK. Email: s.banerjee@bsms.ac.uk

First received 20 May 2012, final revision 10 Oct 2012, accepted 18 Oct 2012

\section{References}

1 Wimo A, Prince M. World Alzheimer's Report 2010 the Global Economic Impact of Dementia. Alzheimer's Disease International, 2010.

2 Comas-Herrera A, Wittenberg R, Pickard L, Knapp M. Cognitive impairment in older people: future demand for long-term care services and the associated costs. Int J Geriatr Psychiatr 2007; 22: 1037-45.

3 Devanand DP, Jacobs DM, Tang MX, Del Castillo-Castaneda C, Sano M, Marder $\mathrm{K}$, et al. The course of psychopathologic features in mild to moderate Alzheimer disease. Arch Gen Psychiatry 1997; 54: 257-63.

4 Lyketsos CG, Lopez O, Jones B, Fitzpatrick AL, Breitner J, De Kosky S. Prevalence of neuropsychiatric symptoms in dementia and mild cognitive impairment: results from the Cardiovascular Health Study. JAMA 2002; 288: 1475-83.

5 Steffens DC, Fisher GG, Langa KM, Potter GG, Plassman BL. Prevalence of depression among older Americans: the Aging, Demographics and Memory Study. Int Psychogeriatr 2009; 21: 879-88.

6 Kales HC, Blow FC, Copeland LA, Bingham RC, Kammerer EE, Mellow AM. Health care utilization by older patients with coexisting dementia and depression. Am J Psychiatry 1999; 156: 550-6.

7 Bains J, Birks JS, Dening TR. The efficacy of antidepressants in the treatment of depression in dementia. Cochrane Database Syst Rev 2002; 4: CD003944.

8 Barrett B, Byford S, Knapp M. Evidence of cost-effective treatments for depression: a systematic review. J Affect Disord 2005; 84: 1-13. 
9 Banerjee S, Hellier J, Dewey M, Romeo R, Ballard C, Baldwin R, et al. Sertraline or mirtazapine for depression in dementia (HTA-SADD): a randomised, multicentre, double-blind, placebo-controlled trial. Lancet 2011 378: 403-11.

10 McKhann G, Drachman D, Folstein M, Katzman R, Price D, Stadlan EM. Clinical diagnosis of Alzheimer's Disease: report of the NINCDS-ADRDA work group. Neurology 1984; 34: 939-44.

11 Alexopoulos GA, Abrams RC, Young RC, Shamoian CA. Cornell Scale for Depression in Dementia. Biol Psychiatr 1988; 23: 271-84.

12 The EuroQoL Group. EuroQoL-a new facility for the measurement of health-related quality of life. Health Policy 1990; 16: 199-208.

13 Dolan P, Gudex C, Kind P, Williams A. A Social Tariff for EuroQol: Results from a UK Population Survey. Discussion Paper 138. University of York 1995.

14 Beecham J, Knapp M. Costing psychiatric interventions. In Measuring Health Needs (2nd edn) (ed. G Thornicroft): 200-24. Gaskell, 2001.

15 Curtis L. Unit Costs of Health and Social Care. PSSRU, 2010.

16 Department of Health. National Health Service Schedule of Reference costs 2010. Department of Health, 2011 (http://www.doh.gov.uk/nhsexec/ refcosts.htm).

17 British Medical Association and Royal Pharmaceutical Society of Great Britain. British National Formulary 59. BMA, RPS 2010.

18 National Institute for Health and Clinical Excellence. Guide to the Methods of Technology Appraisal. NICE, 2008 (http://www.nice.org.uk/media/B52/A7/ TAMethodsGuideUpdatedJune2008.pdf).
19 Knapp M, Thorgrimsen L, Patel A, Spector A, Hallam A, Woods B, et al. Cognitive stimulation therapy for people with dementia: cost-effectiveness analysis. Br J Psychiatry 2006; 188: 574-80.

20 Kendrick T, Chatwin J, Dowrick C, Tylee A, Morriss R, Peveler R, et al. Randomised controlled trial to determine the clinical effectiveness and costeffectiveness of selective serotonin reuptake inhibitors plus supportive care, versus supportive care alone, for mild to moderate depression with somatic symptoms in primary care: the THREAD (THREshold for AntiDepressant response) study. Health Technol Assess 2009; 13: 1-159.

21 Appleby J, Devlin N, Parkin D. NICE's cost-effectiveness threshold: how high should it be? BMJ 2007; 335: 358-9.

22 Schittecatte M, Dumont F, Machowski R, Cornil C, Lavergne F, Wilmotte J. Effects of mirtazapine on sleep polygraphic variables in major depression. Neuropsychobiology 2002; 46: 197-201.

23 Muhlbacher M, Konstantinidis A, Kasper S, Eichberger G, Hinterhuber $\mathrm{H}$ Hofmann $P$, et al. Intravenous mirtazapine is safe and effective in the treatment of depressed inpatients. Neuropsychobiology 2006; 53: 83-87.

24 Naglie G, Tomlinson G, Tansey C, Irvine J, Ritvo P, Black SE, et al. Utilitybased quality of life measures in Alzheimer's disease. Qual Life Res 2006; 15 631-43.

25 Banerjee S. Living well with dementia - development of the National Dementia Strategy for England. Int J Geriatr Psychiatr 2010; 25: 917-25.

26 Banerjee S, Murray J, Foley B, Atkins L, Schneider J, Mann A. Predictors of institutionalisation in older people with dementia. J Neurol Neurosurg Psychiatr 2003; 74: 1315-6.

Martin S. Humphreys

Shoplifting is common. The Centre for Retail Research estimates that customer and staff theft in the UK accounted for nearly $£ 4000$ million in 2011 alone. Generally there is no link with mental disorder. But it can be associated with depression, often in apparently well-adjusted, law-abiding women, in middle or later life and what appears to be a conventional marriage. Characteristically in such cases it occurs in a major high street retailer, is of an unwanted item that the perpetrator possesses the funds to buy, which is removed if not ostentatiously, then with no attempt at concealment. A cry for help? 